\title{
Writer's Cramp in Yaoundé (Cameroon): Series of 5 Cases
}

\author{
Mbonda Paul-Cedric ${ }^{1,}$, , Gams Massi Daniel ${ }^{2}$, Noutsa Laura ${ }^{3}$, Fogang Yannick ${ }^{4}$, \\ Djientcheu Vincent De Paul ${ }^{1}$
}

${ }^{1}$ Faculty of Medicine and Biomedical Sciences, University of Yaoundé I, Yaoundé, Cameroon

${ }^{2}$ Faculty of Medicine and Pharmaceutical Sciences, University of Buea, Buea, Cameroon

${ }^{3}$ Neurology Department, Jamot Hospital in Yaoundé, Yaoundé, Cameroon

${ }^{4}$ Faculty of Medicine and Pharmaceutical Sciences, University of Dschang, Dschang, Cameroon

Email address:

mbondapaul@gmail.com (M. Paul-Cedric)

${ }^{*}$ Corresponding author

\section{To cite this article:}

Mbonda Paul-Cedric, Gams Massi Daniel, Noutsa Laura, Fogang Yannick, Djientcheu Vincent De Paul. Writer's Cramp in Yaoundé (Cameroon): Series of 5 Cases. American Journal of Psychiatry and Neuroscience. Vol. 9, No. 3, 2021, pp. 86-89.

doi: 10.11648/j.ajpn.20210903.11

Received: June 25, 2021; Accepted: July 10, 2021; Published: July 19, 2021

\begin{abstract}
Writer's cramp is focal dystonia, occurring during writing. Its prevalence is very low in the general population and this pathology therefore considered a rare disease. Its diagnosis is clinical but treatment is still a problem in developing countries because botulinum toxin is not yet available. There is little data in sub-Saharan Africa on writer's cramp. We report a series of 5 cases observed at the Yaoundé General Hospital (Cameroon). Methods: We carried out descriptive and prospective work, in which we collected clinical, paraclinical, therapeutic and evolutionary data from patients who came for outpatient consultations for the writer's cramp during 2020 at the Yaoundé General Hospital. Results: The prevalence of this pathology in neurology consultation was $0.29 \%$. These were three women and two men, with an average age of 32.8 years. All had noncontributory personal and family backgrounds. They all received a drug treatment based on trihexyphenidyle, muscle relaxants, anxiolytics, associated with physiotherapy. Baclofen was started in the event of failure on trihexyphenidyl, only one patient received botulinum toxin. The outcome was favorable in a single patient, two-thirds of the patients learned to write with the opposite hand. Conclusion: Writer's cramp is an infrequent condition, very often underdiagnosed. It has a real impact on the quality of life because care is difficult, particularly in our context.
\end{abstract}

Keywords: Writer's Cramp, Dystonia, Yaoundé

\section{Introduction}

Writer's cramp is an abnormal movement manifested by difficulty in writing, which becomes painful and even impossible. It belongs to the dystonia groups [1].

It is a so-called focal dystonia, it affects only one part of the body (the hand or the arm), and also a functional dystonia because it only occurs during the performance of a particular task that is writing. It manifests as abnormal posture and movement of the hand, wrist, fingers, occurring during writing secondary to involuntary contraction of affected muscles, and activation of inappropriate muscles [2].

According to several studies the prevalence of writer's cramp is low (7 to 69 cases per million population) and is therefore considered a rare disease [3].
According to several authors, there is a genetic component in writer's cramp, and in some cases other forms of dystonia are found in a family member of patients who suffer from it [4].

The diagnosis of writer's cramp is clinical and can also be made using the electroneuromyogram, but treatment is still a problem in developing countries because botulinum toxin, which is the gold standard treatment, is not yet accessible. Anticholinergics such as trihexyphenidyl are prescribed with good results in a few cases [5].

There is little data in sub-Saharan Africa on writer's cramp. We report a series of 5 cases observed at the Yaoundé General Hospital (Cameroon). 


\section{Methods}

We report a series of five patients followed in outpatient consultations for the writer's cramp during 2020 at the Yaoundé General Hospital. In this work, which was descriptive and prospective, we collected clinical, therapeutic and evolutionary data from patients who came to consult and follow up for the writer's cramp at the Yaoundé General Hospital.

The Yaoundé General Hospital is a 1st category referral hospital in Cameroon, in Yaoundé the political capital, it has two consultants' neurologists, and patients suffering from pathology of the nervous system can consult there every day.

For all our patients the diagnosis of the writer's cramp was made clinically, in front of each patient who came to consult for disorders or even inability to write without any other associated complaint.

The following examinations were requested from each patient: full blood count, fasting blood sugar, complete blood electrolytes, blood urea and creatinine, and each patient is monitored to date.

Ethics Approval

This work was approved by the Regional Ethics Committee and carried out in accordance with the Declaration of Helsinki of 1964.

\section{Results}

Out of 1,712 patients who came to consult in neurology during the year 2020 at the Yaoundé General Hospital, only five of them suffered from writer's cramp, ie a hospital-based prevalence of $0.29 \%$. These were three women and two men. The average age was 32.8 years. Our patients all had an activity: school, academic or professional. No contributory personal and family history was found (Table 1).

Table 1. Clinical and paraclinical characteristics of patients.

\begin{tabular}{|c|c|c|c|c|c|c|c|}
\hline Items & Age & Sex & Occupation & Medical and familial history & Other complaints & Biological exams & Outcome \\
\hline 1 & 17 & M & Pupil & Non-contributory & No & Normal & Use the other hand \\
\hline 2 & 23 & $\mathrm{~F}$ & Student & Non-contributory & No & Normal & Use the other hand \\
\hline 3 & 42 & $\mathrm{~F}$ & Secretary & Non-contributory & No & Normal & Use the other hand \\
\hline 4 & 38 & M & Computer scientist & Non-contributory & No & Normal & Improvement \\
\hline 5 & 44 & $\mathrm{~F}$ & Accountant & Non-contributory & No & Normal & Lost to follow-up \\
\hline
\end{tabular}

Apart from the writing disorder, the patients did not complain of any other symptoms, the neurological examination was normal. The biological examinations carried out (full blood count, fasting blood glycemia, complete blood electrolytes, blood urea and creatinine) all were normal.

All the patients were put on trihexyphenidyl, with which muscle relaxants and anxiolytics were combined. Baclofen was started for failure on trihexyphenidyl. A patient received a botulinum toxin injection. Physiotherapy was systematic for each patient.

The outcome was marked by learning to write with the opposite hand for $60 \%$ of patients, it was favorable for one patient (20\%) and one patient was lost to follow-up (20\%).

\section{Discussion}

Writer's cramp is considered a rare disease because of its very low prevalence, with 7-69 cases per million inhabitants according to several authors [3]. Our hospital-based prevalence for neurology consultations in 2020 was $0.29 \%$. These results cannot be compared given the different samples, studies in the general population must be carried out to contribute to a better knowledge on the epidemiological level of the writer's cramp in Cameroon.

We found a predominance of the female sex in this series of cases, according to the authors a slight male predominance is observed [6] however some series also find predominance of the female sex [7].

The average age in our series was 32.8 years, the writer's cramp is a disease more described in the young subject [8], this can be explained by the possibility of a genetic sensitivity which could exist, but the later form appears to be the consequence of overuse of writing in subjects with poor gestural habits [9].

All of our patients were right-handed, the literature describes several right-handed people with writer's cramp, but there is no difference in the course of the disease in both right-handed and left-handed [10]. A professional, academic or school activity was found for all the patients in our series, in fact the patients suffering from writer's cramp are described as being very active with their dominant hand, and very often forced to interrupt their activity [8].

We didn't find in our series a family history (for ascendants or collaterals) of writer's cramp for all of our patients. Studies point out that there is a genetic component to writer's cramp [11]. Although several of the cases described are sporadic, a family history of writer's cramp is found in 5 to $20 \%$ of cases [4]. According to some studies, excessive motor activity and a complex writing technique could be the causes of this disease, but this hypothesis remains controversial [12, 13]. Risk factors such as traumatic brain injury, or focal trauma such as excessive motor tasks, sudden increase in daily writing time have also been mentioned as triggering factors for the disease $[14,15]$.

The diagnosis of writer's cramp was made clinically in our series, in front of a focal dystonia occurring during writing with spreading of the elbow, flexion of the wrist or elbow, tightening of the fingers on the object to be held and elevation of 'one or more fingers [16]. Although 
electroneuromyography can help in the writer's cramp diagnosis and especially in management by locating the muscles involved, this exam is not required to make the diagnosis [5]. In addition, functional MRI performed in patients with writer's cramp would reveal gray matter abnormalities [17]. In our context, it is difficult for our patients to perform an electroneuromyogram because it is an expensive examination and functional MRI is not yet available in our country.

Few drugs have been shown to be effective in the treatment of writer's cramp. The proposed treatments aim to strengthen intracortical inhibition. GABA agonists such as benzodiazepines and baclofen have been used [18], as well as anticholinergics under the hypothesis that the pathophysiological mechanisms of the disease are similar to those found in primary dystonias. But the effectiveness of these molecules has yet to be demonstrated. In our series, we noted an improvement in a single patient after various pharmacological tests, associated with functional rehabilitation. Indeed, various motor relearning techniques are proposed and have shown some effectiveness, particularly in combination with other therapies [19]. Botulinum toxin injection is the first-line treatment for focal dystonias and has shown real efficacy in the management of writer's cramp through randomized, doubleblind, placebo-controlled studies. In many patients a longterm normalization of handwriting is reported, but the effect may also be partial [20]. This treatment is not yet available in our country but a patient in our series was able to benefit from it without significant improvement. To date, nearly two-thirds of patients have learned to write with the opposite hand.

\section{Conclusion}

The writer's cramp is an uncommon pathology, it's a focal dystonia occurring during writing whose diagnosis is not very obvious, especially in developing countries in which we have few consultants neurologists. This series of patients living in Yaoundé (Cameroon) shows us that it can also be underdiagnosed and that it has a real impact on the quality of life (several patients stop their activities momentarily for learn to write with the opposite hand). The management is difficult, particularly in our context where botulinum toxin which is the standard treatment is not yet available and the various non pharmacologic approaches (e.g., neurosurgical or neurostimulatory interventions, rehabilitation therapies, adaptive devices) are not or very poorly developed

\section{Acknowledgements}

We are grateful to the staff of Yaoundé General Hospital who helped us in this work. Authors have no financial, political, personal, religious, ideological, academic, intellectual, commercial or any other conflicts of interest to declare in relation to this manuscript.

\section{References}

[1] Jennifer G Goldman. Writer's cramp. 2015; 107: 98-104.

[2] Pearce, J. "A note on scrivener's palsy." J Neurol Neurosurg Psychiatry. 2005 April; 76 (4): 513.

[3] Esde. A prevalence study of primary dystonia in eight European countries. J Neurol. 2000; 247: 787-792.

[4] Bhidayasiri R, Jen JC, Baloh RW. Three brothers with a verylate-onset writer's cramp. Mov Disord. 2005 Oct; 20 (10): 1375-7.

[5] Shavlovskaia OA, Orlova OR, Golubev VL, Veĭn AM. Zh Nevrol Psikhiatr Im S S Korsakova. Clinical and physiological analysis and treatment of writer's cramp 2002; 102 (10): 3541.

[6] Jhunjhunwala K, Lenka A, Pal PK. A Clinical Profile of 125 Patients with Writer's Cramp. Eur Neurol. 2015; 73 (5-6): 316-20.

[7] Snaith A, Wade D. Dystonia. BMJ Clin Evid. 2011; 2011: 1211.

[8] Taira T, Harashima S, Hori T. Neurosurgical treatment for writer's cramp. Acta Neurochir Suppl. 2003; 87: 129-31.

[9] Batla A. Dystonia: A review. Neurol India. 2018; 66 (Supplement): S48-S58.

[10] Jee Hyun Ham, Sang Jin Kim, Sook Keun Song, Chul Hyoung Lyoo, Phil Hyu Lee, Young Ho Sohn, Suk Yun Kang. A prognostic factor in focal hand dystonia: typist's cramp cases and literature review. J Neurol Sci 2016; 371: 85-87.

[11] Siokas V, Aloizou AM, Tsouris Z, Michalopoulou A, Mentis AA, Dardiotis E. Risk Factor Genes in Patients with Dystonia: A Comprehensive Review. Tremor Other Hyperkinet Mov (N Y). 2019; 8: 559.

[12] Rosenkranz K, Williamon A, Butler K, Cordivari C, Lees AJ, Rothwell JC. Pathophysiological differences between musician's dystonia and writer's cramp. Brain. 2005 Apr; 128 (Pt 4): 918-31.

[13] Stahl CM, Frucht SJ. Focal task specific dystonia: a review and update. J Neurol. 2017; 264 (7): 1536-1541.

[14] Roze E, Soumare A, Pironneau I, Sangla S, de Cock VC, Teixeira A, Astorquiza A, Bonnet C, Bleton JP, Vidailhet M, Elbaz A. Case-control study of writer's cramp. Brain. 2009; 132: 756-764

[15] Frei K. J Neurol Sci. Posttraumatic dystonia. 2017; 379: 183191.

[16] Balint B, Mencacci NE, Valente EM, Pisani A, Rothwell J, Jankovic J, Vidailhet M, Bhatia KP. Dystonia. Nat Rev Dis Primers. 2018 Sep 20; 4 (1): 25.

[17] Tomić A, Agosta F, Sarasso E, Svetel M, Kresojević N, Fontana A, Canu E, Petrović I, Kostić VS, Filippi M. Brain Structural Changes in Focal Dystonia-What About Task Specificity? A Multimodal MRI Study. Mov Disord. 2021 Jan; 36 (1): 196-205.

[18] Jankovic J. Treatment of dystonia. Lancet neurology. 2006; 5: 864-872. 
[19] Bleton JP, Vidailhet M, Bourdain F, Ducorps A, Schwartz D, Delmaire C, Lehericy S, Renault B, Garnero L, Meunier S. Somatosensory cortical remodelling after rehabilitation and clinical benefit of in writer's cramp. J Neurol Neurosurg Psychiatry. 2011; 82: 574-577.
[20] Park JE, Shamim EA, Panyakaew P, Mathew P, Toro C, Sackett J, Karp B, Lungu C, Alter K, Wu T, Ahmad OF, Villegas M, Auh S, Hallett $M$. Botulinum toxin and occupational therapy for Writer's cramp. Toxicon. 2019 Nov; 169: $12-17$ 